\title{
Heparin Inhibition of von Willebrand Factor-dependent Platelet Function In Vitro and In Vivo
}

Michael Sobel," Paul M. McNeill, Patricia L. Carison, ${ }^{\star}$ John C. Kermode, Burt Adelman, Regina Conroy, and Dalila Marques Division of Vascular Surgery, Department of Surgery; ${ }^{*}$ Department of Microbiology and Immunology; ${ }^{\ddagger}$ Division of Hematology Oncology, Department of Medicine; Medical College of Virginia, Virginia Commonwealth University; and H. H. McGuire Veterans Affairs Medical Center, Richmond Virginia 23298

\begin{abstract}
The intravenous administration of heparin to patients before open heart surgery reduced ristocetin cofactor activity by $58 \%$ $(P<0.01, t$ test $)$, and this impairment of von Willebrand factordependent platelet function was closely related to plasma heparin levels $\left(r^{2}=0.9\right)$, but not to plasma von Willebrand factor (vWF) levels. We hypothesized that heparin may inhibit vWFdependent platelet hemostatic functions by directly binding $v W F$ in solution and interfering with vWF-GpIb binding. Using the in vitro techniques of ristocetin-induced platelet agglutination, fluorescent flow cytometric measurement of vWF-platelet binding, and conventional radioligand binding assays we observed that heparin inhibited both vWF-dependent platelet function and vWF-platelet binding in a parallel and dose-dependent manner. Heparin also inhibited platelet agglutination induced by bovine vWF and inhibited the binding of human asialo-vWF to platelets in ristocetin-free systems. The inhibitory potency of heparin was not dependent upon its affinity for antithrombin III, but was molecular weight dependent: homogeneous preparations of lower molecular weight were less inhibitory. Heparin impairment of vWF function may explain why some hemorrhagic complications of heparin therapy are not predictable based on techniques for monitoring the conventional anticoagulant effects of heparin. (J. Clin. Invest. 1991. 87:1787-1793.) Key words: binding • ristocetin • platelet aggregation $\cdot$ hemostasis $\cdot$ cardiopulmonary bypass
\end{abstract}

\section{Introduction}

Hemorrhagic complications of heparin anticoagulant therapy remain a significant clinical problem (1-3). Although some studies suggest that bleeding complications may be more common when the anticoagulant effects of heparin are excessive (as judged by coagulation assays), serious bleeding may occur in spite of satisfactory monitoring and control (2). Such hemorrhagic complications may be related to actions of heparin which lie beyond its classic interaction with antithrombin III. Hirsh has raised the possibility that heparin interference with

Portions of this work were previously presented in abstract form at the 1988 National Meeting of the American Federation for Clinical Research. (Clin. Res. 36:420a.)

Address correspondence and reprint requests to Dr. Michael Sobel, Box 108 MCV Station, Richmond, VA 23298.

Received for publication 22 May 1990 and in revised form 20 December 1990.

J. Clin. Invest.

(c) The American Society for Clinical Investigation, Inc. $0021-9738 / 91 / 05 / 1787 / 07 \quad \$ 2.00$

Volume 87, May 1991, 1787-1793 platelet function may be contributory $(1,4)$. Clinical and laboratory investigations have shown that therapeutic doses of heparin can prolong the bleeding time $(5,6)$, and several investigators have noted that heparin in vitro could inhibit ristocetinmediated platelet agglutination (7-9). These findings raise the possibility that heparin's interference with platelet function may involve von Willebrand factor (vWF). ${ }^{1}$ However, little work has been done to elucidate the mechanisms of heparin's action, or to clarify the clinical relevance of heparin's effects on platelet-vWF interactions.

von Willebrand factor plays several crucial roles in platelet hemostasis: at sites of vascular injury vWF anchors platelets to collagen and other exposed subendothelial elements (10-12); at high shear rates vWF may promote platelet aggregation (13, 14). Clinical deficiency of vWF results in a major bleeding diathesis (15). More recently, Ruggeri and others have successfully mapped many of the functional domains of the vWF molecule, providing further insight into the mechanisms by which heparin might actually alter vWF-dependent platelet function $(16,17)$. Von Willebrand factor can bind to two distinct platelet glycoprotein receptors, Ib and IIb/IIIa, via separate binding domains within the protein. In addition, there are specific domains for binding collagen and heparin. The heparin-binding domain overlaps with the GpIb domain in the region from amino acid 449-728 (17). This multifunctionality of a single major interactive region of the protein offers one mechanism for heparin interference with vWF-dependent platelet function.

The goal of this work was to investigate how heparin might impair critical platelet hemostatic functions, specifically via vWF-dependent mechanisms. Studies of clinical patients receiving heparin demonstrated statistically significant inhibition of vWF-dependent platelet function at the time of heparinization. The observations made in heparinized patients were then confirmed by in vitro assays of ristocetin-induced agglutination and vWF-platelet binding in which heparin was added to normal pooled plasma. We have used unfractionated pharmaceutical heparin as well as homogenous heparins to determine the precise dose-response inhibition of vWF-dependent platelet function. Finally, we have demonstrated that heparin inhibition appears to be caused by direct binding of heparin to vWF in solution, rather than heparin binding to the platelet surface or to ristocetin.

\section{Methods}

\section{Reagents}

The following items were purchased: ristocetin, from Bio Data Corp. (Hatboro, PA); anti-von Willebrand factor antibodies, from Atlantic

1. Abbreviations used in this paper: FP, fixed platelets; GpIb, glycoprotein Ib; TBS, tris-buffered saline; vWF, von Willebrand factor 
Antibodies (Scarborough, ME); sodium ${ }^{125}$ I-iodide, from Amersham Corp. (Arlington Heights, IL); Iodobeads and bicinchoninic acid protein assay reagents, from Pierce Chemical Co. (Rockford, IL); ${ }^{3} \mathrm{H}-\mathrm{la}-$ beled porcine mucosal heparin, from New England Nuclear (Boston, MA); unfractionated porcine mucosal heparin (160 USP U/mg, $M_{\text {r }}$ $\sim 12,000$ ), and the remaining reagents from Sigma Chemical Co. (St. Louis, MO). A low molecular weight heparin, RD-3000 (60 USP U/ $\mathrm{mg}, M_{\mathrm{r}} \sim 4,000$ ), was obtained from Calbiochem. Cryoprecipitate was furnished by the Richmond Metropolitan Blood Bank. The following items were generous gifts from collaborating investigators: the monoclonal antibody against platelet GpIb (6D1) from Dr. Barry Coller, State University of New York at Stonybrook; purified bovine von Willebrand factor from Dr. Ed Kirby, Temple University; a number of discrete low molecular weight heparin fragments (including LHN-1 60 USP U/mg, $M_{\mathrm{r}} \sim 6,000$ ) were from Dr. Per Ostergaard of the Novo Research Institute, Denmark. These low molecular weight heparins were all prepared by nitrous acid depolymerization of unfractionated heparins, without other known alterations of chemical structure.

\section{Platelet preparations}

Platelet rich plasma (PRP) was prepared from fresh citrated blood (final concentration of trisodium citrate of $0.38 \%$ ) from normal, medication free donors, by differential centrifugation at $160 \mathrm{~g}$ for $12 \mathrm{~min}$ at room temperature. Formaldehyde fixed platelets (FP) were prepared by fixation of PRP with an equal volume of $2 \%$ buffered formaldehyde for $1 \mathrm{~h}$ at $37^{\circ} \mathrm{C}$; the platelets were washed twice in tris-buffered saline (TBS: $0.05 \mathrm{M}$ Tris, $0.1 \mathrm{M} \mathrm{NaCl}, \mathrm{pH}$ 7.4) and resuspended in the same buffer at a concentration of $400,000 / \mu 1$. For aggregometry and flow cytometry, the FP were diluted 1:1 with normal pooled plasma. Pooled citrated plasma was prepared from 15-20 normal drug free volunteers and stored in aliquots at $-70^{\circ} \mathrm{C}$. Gel filtration of fixed platelets was performed according to Tangen (18) on Sepharose 2B (Pharmacia Fine Chemicals, Piscataway, NJ) in TBS without apyrase.

\section{Platelet aggregometry}

Platelet aggregation in response to ristocetin or purified vWF was measured in a Scienco (Morrison, CO.) two channel aggregometer, as previously described (19). Platelet counts were adjusted to $200,000 / \mu 1$. To study the effects of heparins, samples of fresh PRP or FP in normal pooled plasma were preincubated with the stated concentrations of heparin or TBS buffer control for $10 \mathrm{~min}$ before adding the agonist. Results were expressed as a percentage of the maximal response of control samples (unheparinized $=100 \%$ ), and termed aggregation for PRP and agglutination for FP.

\section{Clinical studies}

Citrated whole blood was obtained from patients about to undergo coronary artery bypass procedures. All gave informed consent according to a protocol approved by the Committee on the Conduct of $\mathrm{Hu}$ man Research at Virginia Commonwealth University. All patients were free of major hemostatic defects and none had significant valvular heart disease. A baseline (control) sample was obtained $24 \mathrm{~h}$ before surgery. Subsequent samples were obtained at designated intervals before and after the administration of intravenous heparin. Ristocetin-induced platelet agglutination was measured at each time point, using washed, pooled fixed platelets from normal donors suspended in the individual patient's platelet poor plasma samples obtained at the appointed intervals. A threshold concentration of ristocetin $(0.75-1.0 \mathrm{~g} /$ liter) was chosen for each patient to produce maximal agglutination: using this constant dose of ristocetin the agglutination response at each timepoint was quantified as a percentage of the control value obtained $24 \mathrm{~h}$ before surgery. Separate aliquots of platelet free plasma were stored at $-70^{\circ} \mathrm{C}$ for later heparin and vWF-antigen assays. The antifactor $\mathrm{Xa}$ anticoagulant activity of heparin in plasma was determined by the chromogenic substrate method of Teien (20) and expressed as units per milliliter of plasma. Plasma vWF levels were determined by Laurell rocket electrophoresis (21).

\section{Fluorescent flow cytometry}

Duplicate suspensions of fixed platelets in plasma were prepared as for aggregometry, incubated for $10 \mathrm{~min}$ at $37^{\circ} \mathrm{C}$ with heparins or buffer control, then exposed to ristocetin $(1.25 \mathrm{mg} / \mathrm{ml})$ for $3 \mathrm{~min}$ without agitation. The suspensions were then refixed with buffered $2 \%$ formaldehyde, washed twice, and stained with a fluorescein labeled anti-human VWF antibody at saturating concentration. The samples were then washed twice, and mean fluorescence measured from 10,000 platelets using an Ortho Diagnostic Systems Inc. (Westwood, MA) 50H fluorescence flow cytometer equipped with a $5 \mathrm{~W}$ argon laser run at $250 \mathrm{~mW}$ power. The sizing gate was adjusted for single platelets by examination of forward and right-angle light scatter signals from control platelets, and daily calibration was performed with fluorescent beads. Specific platelet-vWF binding in the presence of heparin was expressed as a percentage of maximal binding (ristocetin alone) after subtraction of nonspecific binding (no ristocetin). For controls, fluorescent antibodies against a known platelet surface antigen (beta-2 microglobulin) and against an irrelevant antigen (rabbit $\mathrm{IgG}$ ) were also incubated with identically prepared platelets, and mean fluorescence measured per routine. At the standard experimental concentrations (see below) neither preincubation with heparin, ristocetin, nor both together altered the high level of fluorescence observed with anti-beta-2 microglobulin or the low level of fluorescence with anti-rabbit IgG antibody (data not shown).

\section{${ }^{125}$ I-vWF platelet binding assay}

Purification of $v W F$. Human vWF was purified from cryoprecipitate using a modification of the procedures of Newman et al. (22) and Switzer \& McKee (23). In brief, vWF was isolated by a series of ethanol and polyethylene glycol precipitations and by gel filtration on Sepharose CL-4B. Fractions from the column were monitored for ristocetin cofactor activity using fixed platelets, for protein by bicinchoninic acid assay (24) and for purity by reduced 5\% SDS-PAGE (25). Fractions containing the highest specific activity (typically, 100-150 plasma equivalent $\mathrm{U} / \mathrm{mg}$ ) and greatest purity ( $>95 \%$ ) were pooled and stored frozen in aliquots at $-80^{\circ} \mathrm{C}$.

Radioiodination of $\nu W F$. Purified $v W F(10-15 \mu \mathrm{g})$ was radioiodinated with Iodobeads (26), using $100 \mu \mathrm{Ci}$ sodium [ $\left.{ }^{125} \mathrm{I}\right]$ iodide and a reaction time of $30 \mathrm{~min}$ at room temperature. Unreacted $\left.{ }^{[25} \mathrm{I}\right]$ iodide was removed by chromatography on Sephadex G-25 $(1 \times 4-\mathrm{cm}$ column $)$ in $100 \mathrm{mM} \mathrm{NaCl}, 1 \mathrm{mM}$ EDTA, $50 \mathrm{mM}$ Tris- $\mathrm{HCl}, \mathrm{pH} \mathrm{7.5,} \mathrm{containing}$ $0.1 \%$ (wt/vol) BSA (NaCl/EDTA/Tris/BSA). The resulting ${ }^{125}$ I-labeled vWF had an estimated specific radioactivity of $5-7 \mathrm{Ci} / \mathrm{g}$ (corresponding to about one iodine atom per vWF subunit). It was stored in aliquots at $-80^{\circ} \mathrm{C}$ for use within 4 wk. Approximately $50 \%$ of this radioligand was receptor reactive (27), when assessed by a procedure analogous to that used previously for chemotactic formyl peptides (28).

Ristocetin-induced binding of ${ }^{125}$ I-labeled $v W F$ to fixed platelets. ${ }^{125}$ I-Labeled VWF $(\sim 5 \mathrm{ng})$ was incubated in the presence of $1 \mathrm{~g} /$ liter ristocetin with formalin-fixed platelets $(5,000$ cells $/ \mu l$, final concentration) in NaCl/EDTA/Tris/BSA $(200 \mu \mathrm{l})$. The incubation period of $1 \mathrm{~h}$ at $37^{\circ} \mathrm{C}$ was chosen (on the basis of preliminary studies) to ensure equilibration at the concentrations of radioligand and platelets used. The binding reaction was stopped by addition of a further $800 \mu \mathrm{l}$ of cold $\left(4^{\circ} \mathrm{C}\right)$ assay buffer and immediate centrifugation at $15,000 \mathrm{~g}(10 \mathrm{~min}$, $\left.4^{\circ} \mathrm{C}\right)$. The supernatant fraction was aspirated and the ${ }^{125} \mathrm{I}$ content of the pellet then assessed by means of an LKB Produkter (Turku, Finland) CompuGamma 1282 gamma counter. Nonspecific binding (typically $7 \%$ of the added ${ }^{125}$ I-vWF ) was assessed by parallel incubations in the absence of ristocetin; this method yielded estimates of nonspecific binding that were indistinguishable from the traditional method (binding in the presence of excess nonradioactive ligand). Binding data were analyzed using the computer program PREBIND (written in QuickBASIC for the IBM-PC; IBM Instruments, Inc., Danbury, CT).

\section{Other methods}

The direct binding of heparin to platelets was determined as previously described (29). Briefly, washed fresh platelets were incubated with a 
${ }^{3} \mathrm{H}$-labeled unfractionated porcine heparin and centrifuged through silicon oil to separate bound from free heparin.

Heparins with high and low affinity for antithrombin III were prepared by affinity chromatography according to Anderson (30). Molecular weight sizing of all heparins was confirmed in our laboratory by HPLC on a Synchropak GPC100 column using polystyrene sulfonate and heparin standards. Uronic acid content was measured according to Blumenkrantz et al. (31).

Fractionation of $0.1-\mathrm{ml}$ aliquots cryoprecipitate was performed in TBS with $1 \mathrm{mM}$ EDTA on a $1 \times 12$-cm column of Sepharose 4B flowing at $10 \mathrm{ml} / \mathrm{h}$, collecting $0.3-\mathrm{ml}$ fractions. For each fraction, the protein content was determined spectrophotometrically by absorption at $280 \mathrm{~nm}$; ristocetin cofactor (VWF) activity was determined by aggregometry using FP. When chromatography was done in the presence of heparin, a small amount of ${ }^{3}[\mathrm{H}]$ heparin was added, and the heparin concentration in each fraction was detected by scintillation counting.

\section{Results}

\section{Clinical studies of heparinized patients}

12 patients were studied during the administration of intravenous porcine heparin before cardiopulmonary bypass for coronary artery bypass surgery. To establish baseline levels of vWF function, samples of plasma obtained $24 \mathrm{~h}$ preoperatively were tested for vWF activity by measuring ristocetin-induced agglutination of normal FP suspended in the patient's plasma. Subsequent samples were obtained $(a)$ before the induction of anesthesia ("preop"), (b) 15 min before intravenous heparin, and (c) 15 min after heparin. All these samples were obtained before the onset of cardiopulmonary bypass. Later, samples were collected $15 \mathrm{~min}$ before and after protamine administration, and $24 \mathrm{~h}$ postoperatively.

Fig. 1 illustrates the relationship between heparin level and vWF activity. Ristocetin-induced platelet agglutination fell from $120 \%$ of baseline to $50 \%$ of baseline after intravenous heparin (a 58\% decline, $P<0.01$, paired $t$ test). This sudden impairment of vWF-dependent platelet function could not be attributed to major clinical changes in the patient nor to extracorporeal circulation which had not yet begun. There was a close relationship between the mean heparin levels at different stages of the study and the corresponding vWF functional activity $\left(r^{2}=0.9\right.$ by linear regression analysis). These changes in vWF functional activity were not caused by changes in vWF concentration. Throughout the study, mean plasma levels of vWF antigen (measured by rocket electrophoresis) ranged from 99 to $200 \%$ of normal. During the interval of $15 \mathrm{~min}$ before and after heparin, mean vWF levels actually increased from 99 to $126 \%$ of normal, while functional activity fell from 120 to $50 \%$.

\section{In vitro studies}

Ristocetin-induced agglutination. Unfractionated pharmaceutical grade porcine heparin inhibited agglutination in a dosedependent manner. Concentrations of heparin between 42-75 $\mu \mathrm{M}(80-140 \mathrm{U} / \mathrm{ml}$ plasma) completely suppressed ristocetininduced agglutination, while at plasma concentrations encountered clinically $(1-5 \mathrm{U} / \mathrm{ml}, 0.5-2.6 \mu \mathrm{M})$ heparin still produced $10-15 \%$ inhibition. Fig. $2 A$ illustrates a dose-response curve developed from five independent experiments with an unfractionated porcine mucosal heparin $\left(M_{\mathrm{r}} 12,000\right)$. Experiments performed with fresh platelets in plasma gave similar results (data not shown).

Ristocetin-induced $v W F$-platelet binding. Unfractionated heparin also inhibited the binding of vWF to fixed platelets in

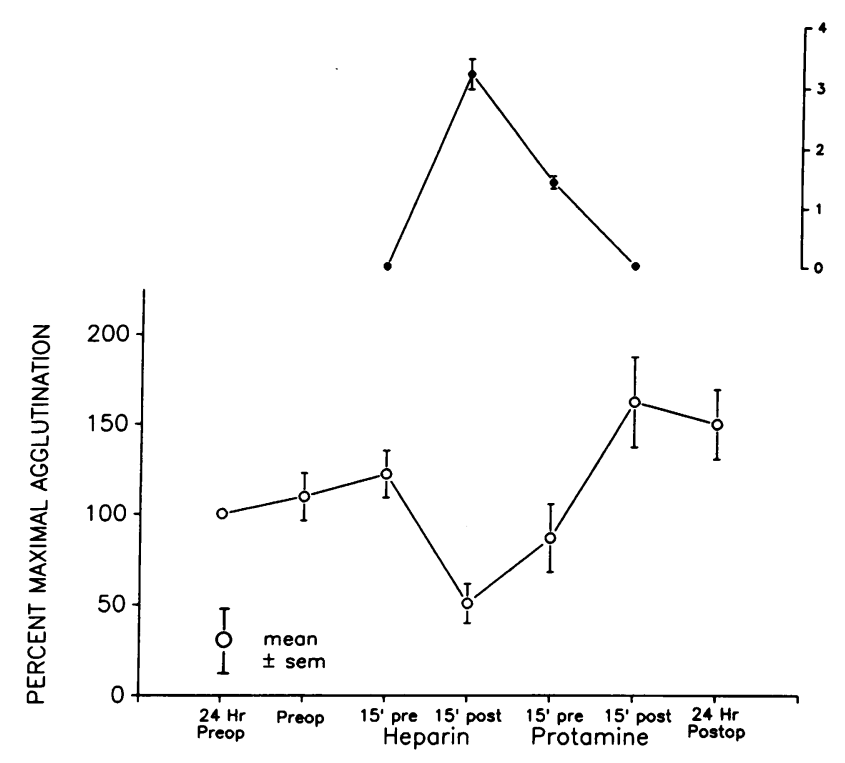

Figure 1. Intravenous heparin inhibits vWF-dependent platelet function. Blood samples were obtained during the period of heparinization for coronary artery bypass grafting. Using normal pooled fixed platelets suspended in patient plasma, ristocetin cofactor activity (agglutination) was expressed as a percentage of the baseline VWF activity in the 24-h preoperative sample. The concentration of heparin in the same plasma samples is expressed as anti-Xa $\mathrm{U} / \mathrm{ml}$ plasma. Statistical significance was determined by the paired $t$ test: platelet agglutination fell significantly after heparin (pre- versus post-heparin, $P<0.01$ ), and rose significantly after protamine (pre- versus post-protamine $P$ $<0.01$ ). There were no statistically significant differences between the platelet function at the "preop" or "pre-heparin" point compared with the normal baseline $24 \mathrm{~h}$ preoperatively.

the presence of ristocetin. Using fluorescent flow cytometry of platelets in plasma, a concentration-dependent inhibition of binding was observed (Fig. $2 \mathrm{~B}$ ). Both the agglutination assay and the cytometer binding studies demonstrated a similar $\mathrm{IC}_{50}$ (concentration producing $50 \%$ inhibition) in a range of $10-30$ $\mu \mathrm{M}$ for unfractionated heparin $\left(M_{\mathrm{r}} 12,000\right)$ in the normal plasma milieu. In fact, the entire dose-response data for inhibition of agglutination and inhibition of binding by cytometry were closely correlated $\left(r^{2}=0.83\right.$, linear regression analysis of all data points for $M_{\mathrm{r}} 12,000$ heparin in Figs. $2 A$ and $B$ ).

To further elucidate the inhibitory action of heparin on platelet-vWF binding we then performed binding experiments in an isolated plasma free system using fixed washed platelets, ristocetin, and purified ${ }^{125} \mathrm{I}$-labeled $\mathrm{vWF}(25 \mathrm{ng} / \mathrm{ml})$ at concentrations below the anticipated $K_{\mathrm{d}}$ for heparin-vWF interaction. These studies (Fig. $2 C$ ) show the same inhibitory pattern of dose response and molecular weight dependency that was observed in the agglutination and cytometric assays, although the $\mathrm{IC}_{50}$ for unfractionated heparin is much lower in the isolated radioligand assay system $(\sim 5 \mathrm{nM})$.

We hypothesized that the discrepancy between the different IC $_{\text {so }}$ 's was due to: $(a)$ a reduction in the free heparin concentration by heparin binding proteins present in plasma, and $(b)$ inflation of the apparent $\mathrm{IC}_{50}$ in the agglutination and flow cytometric studies by the relatively large excess of VWF present in plasma ( $\sim 1,000$-fold higher than in the radioligand assay). Therefore we restudied vWF-dependent platelet agglutination and its inhibition by heparin using a simplified system resem- 

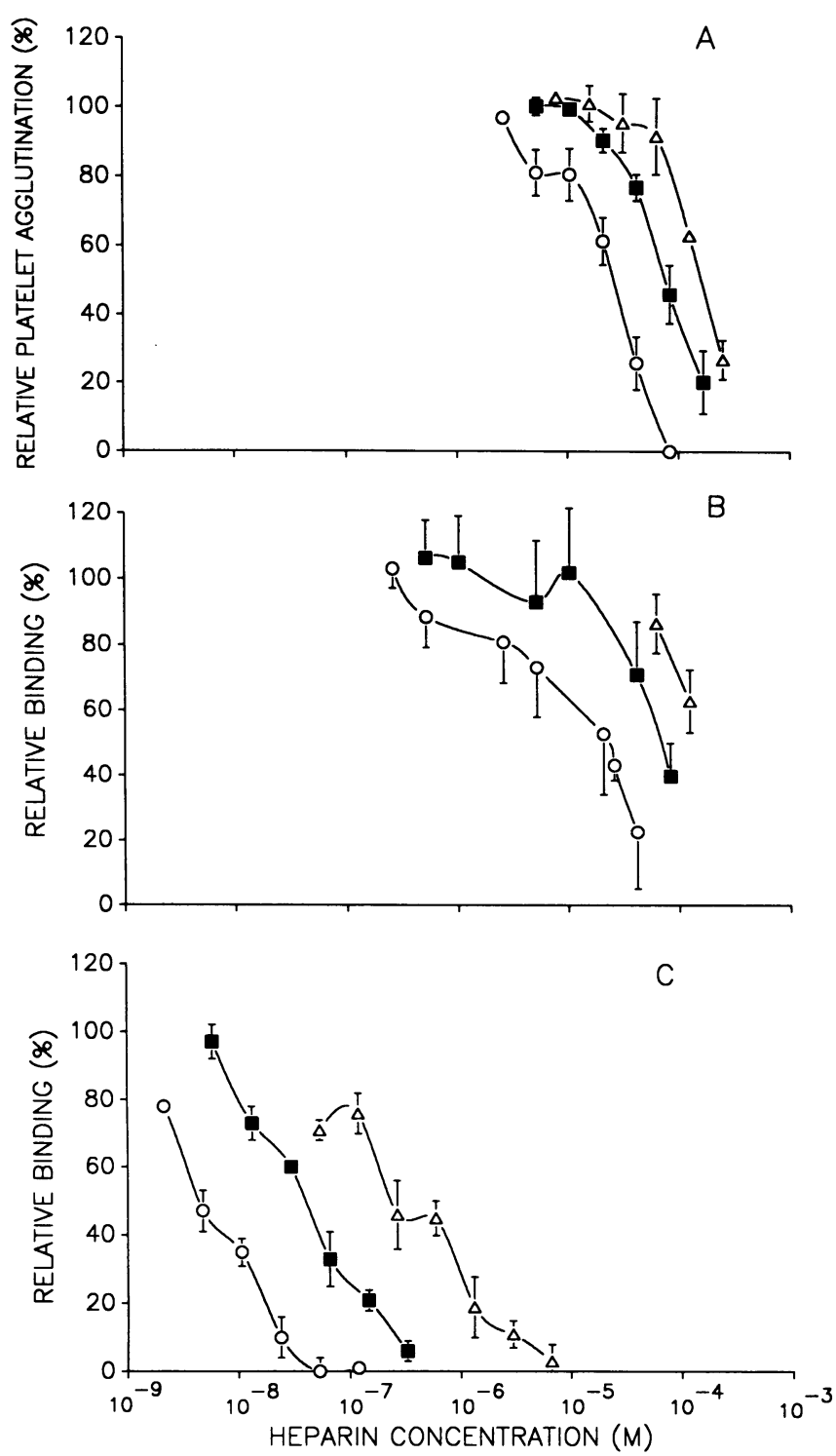

Figure 2. In vitro inhibition by heparins of vWF-dependent platelet agglutination and vWF-platelet binding. These data represent the means and SEM of three to five independent experiments. Fixed platelets were incubated with heparins of different molecular weights ([- - - ] molecular weight 12,$000 ;[-\square-] 6,000 ;[-\Delta-] 4,000$ ) and concentration, and then binding or agglutination was measured in response to ristocetin $1 \mathrm{~g} /$ /iter. See text for details of experimental methods. $(A)$ Ristocetin-induced agglutination of fixed platelets suspended in plasma was inhibited in a dose- and molecular weight-dependent manner by heparins of three molecular weight ranges. $(B)$ Measurement of platelet-vWF binding in plasma by fluorescent flow cytometry, using the same heparins. $(C)$ Measurement of plateletvWF binding by conventional ${ }^{125} \mathrm{I}-\mathrm{vWF}$ binding assay in buffer, using the same heparins as above.

bling that of the binding assay: fixed washed platelets in buffer, ristocetin, and the minimal concentrations of purified vWF necessary for agglutination. As expected, the observed $\mathrm{IC}_{50}$ for inhibition of agglutination by unfractionated heparin was over $1,000$-fold lower ( $20 \mathrm{nM})$ in this assay system, compared with the $\mathrm{IC}_{50}$ of heparin in plasma $(30 \mu \mathrm{M})$.

Comparison of heparin fractions. Fig. 2 illustrates that heparin fractions of lower molecular weight $\left(M_{\mathrm{r}} 6,000\right.$ and 4,000)
Table I. Inhibition by High and Low Antithrombin Affinity Heparin

\begin{tabular}{cc}
\hline Heparin type & $\begin{array}{c}\text { Mean } \pm \text { SEM } \\
\text { vWF bound }\end{array}$ \\
\hline $0.25 \mathrm{mg} / \mathrm{ml}$ & $\%$ of $\max$ \\
Unfractionated & $56.8 \pm 7.8$ \\
High AT-III affinity & $63.9 \pm 6.0$ \\
Low AT-III affinity & $57.8 \pm 7.8$
\end{tabular}

vWF-platelet binding induced by ristocetin was measured by fluorescent flow cytometry as described in the text. The data summarize four independent experiments performed in duplicate. The inhibitory potency of heparins with high or low affinity for antithrombin III (AT-III) were not significantly different from the unfractionated original heparin $(P>0.05, t$ test $)$.

were respectively less inhibitory. The same motif of relative potency was observed in agglutination experiments, or binding measured by flow cytometry or radioligand assay. These molecular weight differences were apparent, whether the polysaccharides were compared by weight or on a molar basis. We chromatographed porcine mucosal heparin on insolubilized antithrombin III into fractions with high $(>600 \mathrm{U} / \mathrm{mg})$ and low $(<50 \mathrm{U} / \mathrm{mg})$ anti-factor $\mathrm{Xa}$ anticoagulant activity. These fractions were otherwise similar in molecular weight range and net charge as judged by GPC HPLC and isoelectric focussing. Table I demonstrates that both high and low antithrombin affinity heparins were equally potent inhibitors of platelet-vWF binding as unfractionated heparin.

\section{In vitro studies without ristocetin}

The effects of bovine and asialo-human $v W F$. Because a direct interaction between heparin and ristocetin might also explain heparin's inhibitory effects, agglutination experiments were performed with bovine vWF. Bovine vWF binds spontaneously to platelet glycoprotein $\mathrm{Ib}$, and does not require ristocetin as a cofactor for binding or agglutination (7). Fixed washed platelets were agglutinated with bovine vWF $(20 \mu \mathrm{g} / \mathrm{ml})$ purified according to Kirby (7). Preincubation with heparin produced inhibition comparable to that observed for inhibition of ristocetin-induced agglutination of human platelets. The inhibitory potency of heparin was also molecular weight dependent (Fig. 3). The inhibitory effect of heparin could be overcome by increasing the concentration of bovine VWF (data not shown).

When the sialic acid residues of human vWF are removed, von Willebrand factor binds to platelets spontaneously in the

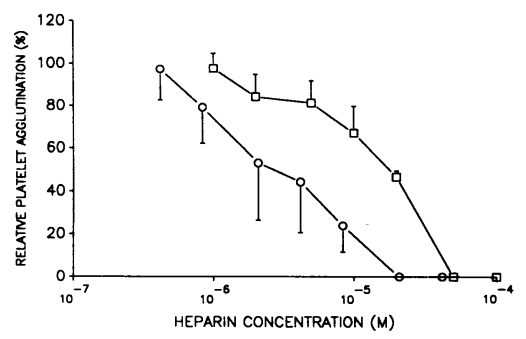

Figure 3. Heparin inhibition of bovine von Willebrand factor. Formaldehyde fixed washed platelets resuspended in TBS with $1 \%$ BSA were directly agglutinated with purified bovine vWF $(20 \mu \mathrm{g} / \mathrm{ml})$ after preincubation with a range of concentrations of an unfractionated or low molecular weight heparin. ([-० - ] molecular weight 12,000 ; [ $\square-] 6,000)$ The data represent the means and SEM of three independent experiments. 
absence of ristocetin. If the inhibitory effects of heparin were independent of ristocetin, we might expect heparin to also inhibit the binding of asialo-vWF to platelets. Therefore, sialic acid residues were removed from purified human von Willebrand factor by neuraminidase digestion as described by DeMarco and Shapiro (32). To determine the effects of heparin on asialo-vWF-platelet binding, pairs of fixed platelet suspensions were mixed with asialo-vWF in the presence and absence of heparin $(21 \mu \mathrm{M}, 40 \mathrm{U} / \mathrm{ml})$ and vWF-platelet binding was measured by flow cytometry as previously described. Heparin reduced asialo vWF-platelet binding by $54 \pm 4 \%$ (mean \pm SD of two independent experiments).

Heparin-platelet binding. Heparin's inhibition of vWF binding might result from heparin bound directly to glycoprotein $\mathrm{Ib}$ (GpIb), the principal vWF receptor. To determine whether heparin was binding quantitatively to $\mathrm{GpIb}$, we specifically blocked the receptor with monoclonal antibody 6D1 (33), and then measured heparin-platelet binding (29). Antibody blockade of GpIb should reduce heparin binding, if heparin binds significantly to this receptor. At concentrations that completely suppressed ristocetin induced agglutination, there was no significant change in the amount of heparin that bound to the platelet ( $109 \%$ of control binding $\pm 2.9 \%$, mean \pm SEM of three independent assays).

Direct evidence of heparin-von Willebrand factor binding. Cryoprecipitate, heparinized cryoprecipitate $(0.83 \mathrm{mM}, 10$ $\mathrm{mg} / \mathrm{ml}$ ), and heparin alone were sequentially passed over a Sepharose 4B column. The goals were to determine: $(a)$ whether heparin would comigrate with the protein fraction containing von Willebrand factor, and if so, $(b)$ did the heparin bound to vWF in solution impair the ristocetin cofactor activity of those fractions? Fig. 4 summarizes these experiments. Ristocetin cofactor activity was limited to two to four fractions at the front of the void volume, as would be expected for the high molecular weight multimers of vWF. When heparin alone

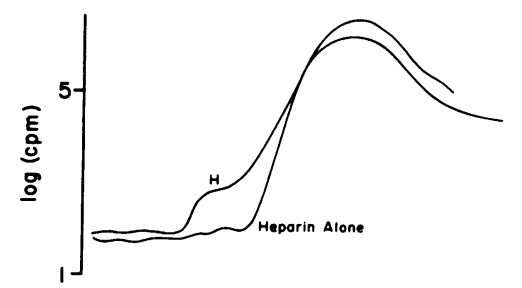

Figure 4. Heparin-vWF binding in solution and the consequent impairment of vWF function. As described in the text, cryoprecipitate alone $(C)$, cryoprecipitate premixed with $\left[{ }^{3} \mathrm{H}\right]$ heparin $(H)$, and heparin alone were serially passed over a Sepharose 4B column. Fractions were analyzed for heparin concentration (top), protein concentration (middle), and ristocetin cofactor activity (bottom). When heparin alone was chromatographed, it eluted as a late broad peak. Chromatography of the cryoprecipitate/heparin mixture $(H)$ revealed that a portion of the

heparin comigrated with the void fraction containing vWF activity. Those fractions demonstrated reduced vWF activity when heparin was present $(\mathrm{C}$ versus $\mathrm{H})$. was applied to the column, it eluted in one broad later peak. However, when heparin mixed with cryoprecipitate was applied, a small portion of the heparin comigrated with the fractions containing von Willebrand factor activity. The ristocetin cofactor activity of those heparin/vWF containing fractions was reduced by an average of 56\% (range 20-100\%; see Fig. 4) compared with identical fractions obtained from the passage of cryoprecipitate alone.

\section{Discussion}

In this investigation we have demonstrated that there is signifcant impairment of von Willebrand factor function in the plasma of acutely heparinized patients. We have characterized the dose-response relationship of heparin inhibition of vWFdependent platelet function, and demonstrated a close correlation between inhibition of function (agglutination) and inhibition of vWF-platelet binding. Finally, we investigated the mechanism of heparin inhibition of vWF function, demonstrating that: $(a)$ heparin's effects were independent of ristocetin and $(b)$ heparin most likely acted not at the platelet surface but in solution by binding vWF and impairing the capacity of vWF to bind the platelet.

Flow cytometry has been successfully used to measure a number of different platelet-ligand interactions, including the binding of plasminogen (34), fibronectin (35), and von Willebrand factor (36); precise quantitative methods have been developed (37). To complement and confirm the validity of our own cytometric binding studies, we also performed classical radioligand binding studies with ${ }^{125} \mathrm{I}-\mathrm{vWF}$ and fixed platelets in a plasma-free system. A similar concentration-dependent inhibition of binding by heparin was observed, but at 1000 -fold lower concentrations of heparin. While these studies performed in an isolated, purified system are most useful in exploring the mechanisms and affinity of the interactions between vWF and heparin, they may not accurately reflect the physiologic state of affairs where there is likely a large functional excess of vWF relative to heparin, and free heparin concentrations may be reduced by binding to other plasma proteins (38). In addition, purified preparations of vWF may not precisely reflect the same heparin-binding properties of native vWF in plasma. The different binding methods (cytometric versus radioligand) point out the advantages of the flow cytometric method to this study: experiments can be performed in plasma using native ligand, and the results may be more directly applicable to in vivo phenomena.

In vitro, the inhibitory effects of an unfractionated pharmaceutical grade of heparin were more potent than those of low molecular weight heparin fragments prepared by nitrous acid depolymerization. To analyze the stoichiometry of the action of the different heparins, they were compared on a molar basis rather than by weight. However, inhibitory potency appeared to be related to polysaccharide chain length, even when compared on the basis of weight. If there is a specific polysaccharide structure or sequence which favors binding vWF, it does not appear to resemble the antithrombin III-binding pentasaccharide, because both high and low antithrombin affinity heparins showed comparable inhibition of vWF function. The results of the cryoprecipitate chromatography experiments (Fig. 4) suggest that there could be a unique subset of heparin molecules with higher vWF affinity.

Because heparin and ristocetin are highly anionic and cat- 
ionic, respectively, we performed experiments in the absence of ristocetin to confirm the observed inhibitory effects of heparin. Using fluorescent flow cytometry, we found that the spontaneous binding of human asialo-von Willebrand factor to platelets was inhibited by heparin. Likewise, the agglutination of platelets by bovine vWF was inhibited by heparin in a manner parallel to the inhibition observed in the human plasma/ristocetin system. The heparin inhibition could be overcome by increasing concentrations of bovine vWF, suggesting that the principal interaction occurred in solution between vWF and heparin, not on the platelet surface.

The radiolabeled vWF experiments illustrated in Fig. $2 C$ demonstrated a major inhibitory effect by heparin at concentrations in the nanomolar range. Our previous studies of heparin-platelet binding (29) have shown that less than $1 \%$ of the total heparin binding capacity of platelets is reached at such concentrations, representing, at most, 1,000 heparin molecules bound per platelet. This quantity of platelet-bound heparin would be impossible to saturate the available replicates of $\mathrm{GpIb}$ ( 26,000-30,000 [39]). Thus, platelet-bound heparin is unlikely to account for the high degree of inhibition of vWF binding by heparin seen at these low concentrations. Blockade of GpIb with a monoclonal antibody did not reduce the quantity of heparin bound to the platelet either. These data all suggest that platelet-bound heparin is unlikely to account for most of the heparin inhibition of platelet-vWF interactions we have observed.

Fractionation of heparin-cryoprecipitate mixtures showed that a portion of heparin comigrated with high molecular weight proteins. These could have been heparin-fibrinogen-fibronectin complexes rather than vWF (40). Yet these same fractions did possess ristocetin cofactor activity, which was reduced in the presence of heparin, suggesting that at least some of the protein was vWF. We are currently characterizing the $K_{\mathrm{d}}$ of heparin-von Willebrand factor binding directly, and exploring the specific heparin-binding domains of vWF (41).

Heparin inhibition of agglutination appeared to be greater in vivo than in vitro. The plasma of acutely heparinized patients showed a $58 \%$ reduction in vWF activity (3-4 U/ml, 1.5-2.0 $\mu \mathrm{M}$ ), while comparable concentrations of heparin in vitro produced $10-15 \%$ inhibition. These differences could not be attributed to changes or differences in the concentration of vWF antigen. In vitro, the pooled plasma contained a consistent amount of von Willebrand factor. In vivo, plasma vWF concentrations were measured to be higher than normal and rose further at the time of heparin administration. This discrepancy in heparin's in vitro versus in vivo effects may be an artifact of our methods for measuring heparin. For the clinical patients, heparin concentrations in plasma were measured by bioassay, while for the in vitro experiments heparin concentrations were determined empirically by the known amount added to plasma. The second method may overestimate the actual amount of free heparin available to bind von Willebrand factor, due to heparin binding to other plasma proteins such as antithrombin III, fibronectin, and histidine rich glycoprotein (38). This explanation is further supported by the radioligand binding assays and agglutination experiments which showed even greater heparin potency in a plasma-free medium.

This specific clinical model was chosen for study due to the consistency and reproducibility of heparinization of a patient population without major preexisting pathologic thromboses or hemorrhagic diathesis. Historically, Pekcelen et al. first suggested that heparin might be responsible for the impairment of vWF function observed during bypass cardiopulmonary (42). Weinstein and colleagues, in their study of the kinetics of vWFantigen levels during cardiopulmonary bypass, observed as we did that vWF levels generally rose to supranormal levels at the conclusion of bypass and in the early postoperative period. They found that the group of patients with lower initial levels of $\mathrm{vWF}$ and smaller postoperative increases suffered greater perioperative blood loss (43).

Although heparin is ultimately neutralized with protamine after open heart surgery, our current data still have relevance for the more common clinical setting where heparin is not neutralized and therapy is longer term (e.g., for venous thromboembolism). Without protamine neutralization, heparin inhibition of vWF-dependent hemostasis may be even more clinically significant even though the absolute doses of heparin are lower. The dramatic inhibition we observed with a single intravenous injection of heparin is in keeping with early observations that intermittent intravenous injection of heparin resulted in more bleeding complications (2). But even continuous intravenous infusion may cause hemorrhage in spite of adequate monitoring if patients have diminished plasma levels of $\mathrm{vWF}$, or possess a range of lower molecular weight multimers. Because pharmaceutical heparins are heterogenous, the anti-vWF effects of different preparations must also be considered.

Several preliminary conclusions can be made about the structure-activity relations of the heparin-vWF interaction. Heparin fragments of lower molecular weight showed less inhibitory potency; high and low antithrombin affinity heparins were equivalent. These observations do not rule out the possibility that there is some polysaccharide structure with higher affinity for vWF. Separation of heparin and vWF on Sepharose $4 \mathrm{~B}$ suggested that there could be a subfraction of heparin molecules with specific vWF-binding affinity. We are currently investigating the identity of this heparin subfraction. Based on the structure-activity maps of von Willebrand factor proposed by Mohri $(17,44)$ and Sixma (45), the domain of vWF responsible for binding platelet GpIb is coincident with an important heparin-binding region. We have recently characterized this heparin-binding domain even more precisely (41). It is feasible that heparin bound to vWF could produce steric hindrance or alter the conformation of the molecule sufficiently to impair vWF binding to GpIb. Heparins of longer chain length may be more effective in exerting their effects on vWF, or may possess more subunits that bind vWF.

The unique effects of heparin on von Willebrand factor function which we have observed open a potentially broad area for further investigation and therapeutic application. Additional study is needed to elucidate the specific structural determinants of heparin's affinity for vWF. The stoichiometry of heparin-vWF binding and the influence of multimeric size must also be determined. Ultimately, new heparins could be developed for clinical use which are effective plasma anticoagulants but lack the hemorrhagic side effects which stem from interference with vWF function. Conversely, heparins with potent anti-vWF activity might be useful where there are areas of pathologic high shear stress (e.g., coronary artery stenoses) where von Willebrand factor-mediated platelet aggregation or adhesion may be prominent (14). 


\section{Acknowledgments}

We are very grateful for the advice and assistance of Dr. Ed Kirby.

Dr. Sobel was supported in this work by National Institutes of Health grant HL-39903 and by the Veterans Administration Research Program.

\section{References}

1. Levine, M. N., and J. Hirsh. 1986. Hemorrhagic complications of anticoagulant therapy. Semin. Thromb. Hemostasis. 12:39-57.

2. Salzman, E. W., D. Deykin, R. M. Shapiro, and R. D. Rosenberg. 1975. Management of heparin therapy. Controlled prospective trial. N. Engl. J. Med. 292:1046-1050.

3. Holm, H. A., U. Abildgaard, and S. Kalvenes. 1985. Heparin assays and bleeding complications in treatment of deep venous thrombosis with particular reference to retroperitoneal bleeding. Thromb. Haemostasis. 54:278-281.

4. Hirsh, J., F. Ofosu, and M. Buchanan. 1985. Rationale behind the development of low molecular weight heparin derivatives. Semin. Thromb. Hemostasis. 11:13-16.

5. Buchanan, M. R., P. N'guyen, J. Van Ryn, F. A. Ofosu, and J. Hirsh. 1986. DDAVP reduces the platelet defect associated with heparin. Circulation. 74:II95.

6. Heiden, D., C. H. Mielke, Jr., and R. Rodvien. 1977. Impairment by heparin of primary haemostasis and platelet [14C]5-hydroxytryptamine release. Br. J. Haematol. 36:427-436.

7. Kirby, E. P., and D. C. B. Mills. 1975. The interaction of bovine factor VIII with human platelets. J. Clin. Invest. 56:491-502.

8. Suzuki, K., J. Nishioka, and S. Hashimoto. 1979. Inhibition of factor VIIIassociated platelet aggregation by heparin and dextran sulfate, and its mechanism. Biochim. Biophys. Acta. 585:416-426.

9. Girolami, A., L. De Marco, L. Virgolini, R. Peruffo, and F. Fabris. 1975. The effect of heparin on platelet aggregation by common inductors and ristocetin in congenital bleeding disorders due to factor VIII or fibrinogen defects. Blut. 31:219-226.

10. Moroose, R., and L. W. Hoyer. 1986. von Willebrand factor and platelet function. Annu. Rev. Med. 37:157-163.

11. Badimon, L., J. J. Badimon, J. Rand, V. T. Turitto, and V. Fuster. 1987. Platelet deposition on von Willebrand factor-deficient vessels. Extracorporeal perfusion studies in swine with von Willebrand's disease using native and heparinized blood. J. Lab. Clin. Med. 110:634-647.

12. McPherson, J., S. Brownlea, and M. B. Zucker. 1987. Effect of monoclonal antibodies against von Willebrand factor and platelet glycoproteins IIb/IIIa on the platelet retention test. Blood. 70:546-550.

13. Moake, J. L. 1989. Insolubilized von Willebrand factor and the initial events in hemostasis. J. Lab. Clin. Med. 114:1-3.

14. Peterson, D. M., N. A. Stathopoulos, T. D. Giorgio, J. D. Hellums, and J. L. Moake. 1987. Shear-induced platelet aggregation requires von Willebrand factor and platelet membrane glycoproteins Ib and IIb-IIIa. Blood. 69:625-628.

15. Ruggeri, Z. M., and T. S. Zimmerman. 1987. von Willebrand factor and von Willebrand disease. Blood 70:895-904.

16. Fujimura, Y., K. Titani, L. Z. Holland, J. R. Roberts, P. Kostel, Z. M Ruggeri, and T. S. Zimmerman. 1987. A heparin-binding domain of human von Willebrand factor. Characterization and localization to a tryptic fragment extending from amino acid residue Val-449 to Lys-728. J. Biol. Chem. 262:1734 1739.

17. Mohri, H., A. Yoshioka, T. S. Zimmerman, and Z. M. Ruggeri. 1989. Isolation of the von Willebrand factor domain interacting with platelet glycoprotein Ib, heparin, and collagen and characterization of its three distinct functional sites. J. Biol. Chem. 264:17361-17367.

18. Tangen, O., H. J. Berman, and P. Marfey. 1971. Gel filtration: a new technique for separation of blood platelets from plasma. Thromb. Diath. Haemorrh. 25:268-278.

19. Kelton, J. G., J. Bishop, C. J. Carter, and J. Hirsh. 1980. A comparison of the quantitative ristocetin von Willebrand factor assay by using fresh and fixed platelets. Thromb. Res. 18:477-483.

20. Teien, A. N., and M. Lie. 1977. Evaluation of an amidolytic heparin assay method: Increased sensitivity by adding purified antithrombin III. Thromb. Res. 10:399-410.

21. Laurell, C. B. 1965. Antigen-antibody crossed electrophoresis. Anal. Biochem. 10:358-362.

22. Newman, J., A. J. Johnson, M. H. Karpatkin, and S. Puszkin. 1971 Methods for the production of clinically effective intermediate- and high-purity factor-VIII concentrations. Br. J. Haematol. 21:1-20.

23. Switzer, M. E., and P. A. McKee. 1976. Studies on human antihemophilic factor: evidence for a covalently linked subunit structure. J. Clin. Invest. 57:925937.

24. Smith, P. K., R. I. Krohn, G. T. Hermanson, A. K. Mallia, F. H. Gartner, M. D. Provenzano, E. K. Fujimoto, N. M. Goeke, B. J. Olson, and D. C. Klenk 1985. Measurement of protein using bicinchoninic acid. Anal. Biochem. 150:7685.

25. Laemmli, U. K. 1970. Cleavage of structural proteins during the assembly of the head of bacteriophage T4. Nature (Lond.). 227:682-690.

26. Markwell, M. A. K. 1982. A new solid-state reagent to iodinate proteins: I. Conditions for the effective labeling of antiserum. Anal. Biochem. 125:427-432.

27. Kermode, J. C. 1989. The curvilinear Scatchard plot: experimental artifact or receptor heterogeniety? Biochem. Pharmacol. 38:2053-2060.

28. Kermode, J. C. 1988. Means for assessment of radioligand quality and its importance in receptor-binding studies: observations with radiolabelled formylmethionyl-leucylphenylalanine. Biochem. J. 252:521-528.

29. Sobel, M., and B. Adelman. 1988. Characterization of platelet binding of heparins and other glycosaminoglycans. Thromb. Res. 50:815-826.

30. Andersson, L.-O., T. W. Barrowcliffe, E. Holmer, E. A. Johnson, and G. E. C. Sims. 1976. Anticoagulant properties of heparin fractionated by affinity chromatography on matrix bound antithrombin III by gelfiltration. Thromb. Res. 9:575-583.

31. Blumenkrantz, N., and G. Asboe-Hansen. 1973. New method for quantiative determination of uronic acids. Anal. Biochem. 54:484-489.

32. DeMarco, L., and S. S. Shapiro. 1981. Properties of human asialo-factor VIII. A ristocetin-independent platelet-aggregating agent. J. Clin. Invest. 78:321328.

33. Coller, B. S., E. I. Peerschke, L. E. Scudder, and C. A. Sullivan. 1983. Studies with a murine monoclonal antibody that abolishes ristocetin-induced binding of von Willebrand factor to platelets: additional evidence in support of gpIb as a platelet receptor for von Willebrand factor. Blood. 61:99-110.

34. Adelman, B., A. Rizk, and E. Hanners. 1988. Plasminogen interactions with platelets in plasma. Blood. 72:1530-1535.

35. Ejim, O. S., M. J. Powling, P. Dandona, P. B. A. Kernoff, and A. H Goodall. 1990. A flow cytometric analysis of fibronectin binding to platelets from patients with peripheral vascular disease. Thromb. Res. 58:519-524.

36. Adelman, B., P. Carlson, and P. Powers. 1987. von Willebrand factor is present on the surface of platelets stimulated in plasma by ADP. Blood. 70:1362 1366.

37. Poncelet, P., and P. Carayon. 1985. Cytofluorometric quantification of cell-surface antigens by indirect immunofluorescence using monoclonal antibodies. J. Immunol. Methods. 85:65-74.

38. Lijnen, H. R., M. Hoylaerts, and D. Collen. 1983. Heparin binding properties of human histidine-rich glycoprotein. J. Biol. Chem. 258:3803-3808.

39. Berndt, M. C., and J. P. Caen. 1984. Platelet Glycoproteins. Prog. Hemo stasis. Thromb. 5:111-150.

40. Menitove, J. E., D. L. Amrani, D. Meh, M. Frenzke, and M. W. Mosesson. 1987. Evaluation of factor VIII-rich cryoprecipitate and the plasma fibronectinrich, heparin-precipitable fraction prepared from single-donor plasma units. Transfusion. 27:491-495.

41. Sobel, M., and R. B. Harris. 1990. Identification of the heparin-binding sequence of human von Willebrand factor. Circulation. 82(Suppl):594.

42. Pekcelen, Y., and S. Inceman. 1975. Letter: heparin and ristocetin-induced platelet aggregation. Br. Med. J. 4:101-102.

43. Weinstein, M., J. A. Ware, J. Troll, and E. Salzman. 1988. Changes in von Willebrand factor during cardiac surgery: effect of desmopressin acetate. Blood. 71:1648-1655.

44. Mohri, H., Y. Fujimura, M. Shima, A. Yoshioka, R. A. Houghten, Z. M Ruggeri, and T. S. Zimmerman. 1988. Structure of the von Willebrand factor domain interacting with glycoprotein Ib. J. Biol. Chem. 263:17901-17904.

45. Girma, J. P., D. Meyer, C. L. Verweij, H. Pannekoek, and J. J. Sixma. 1987. Structure-function relationship of human von Willebrand factor. Blood. 70:605-611. 